\title{
DESTRUCTION OF VERY SIMPLE TREES
}

\author{
JAMES ALLEN FILL, NEVIN KAPUR, AND ALOIS PANHOLZER
}

\begin{abstract}
We consider the total cost of cutting down a random rooted tree chosen from a family of so-called very simple trees (which include ordered trees, $d$-ary trees, and Cayley trees); these form a subfamily of simply generated trees. At each stage of the process an edge is chose at random from the tree and cut, separating the tree into two components. In the one-sided variant of the process the component not containing the root is discarded, whereas in the two-sided variant both components are kept. The process ends when no edges remain for cutting. The cost of cutting an edge from a tree of size $n$ is assumed to be $n^{\alpha}$. Using singularity analysis and the method of moments, we derive the limiting distribution of the total cost accrued in both variants of this process. A salient feature of the limiting distributions obtained (after normalizing in a family-specific manner) is that they only depend on $\alpha$.
\end{abstract}

\section{INTRODUCTION}

Consider the following process on a rooted tree with $n$ vertices. Pick an edge uniformly at random and "cut" it, separating the tree into a pair of rooted trees; the tree containing the root of the original tree retains its root while the tree not containing the root of the original tree is rooted at the vertex adjacent to the edge that was cut. In the one-sided variant of the problem the tree not containing the original root is discarded and the process is continued recursively until the original root is isolated. In the two-sided variant the process is continued recursively on each of the rooted trees. Assume that the cost incurred for selecting an edge and splitting the tree is $t_{n}$. In this paper we derive the limiting distribution of the total cost accrued when the tree is a random very simple tree (defined below) and $t_{n}=n^{\alpha}$ for fixed $\alpha \geq 0$, for both the two-sided variant (Theorems 4.4 4.6 and 4.7) and the one-sided variant (Theorem 5.11). A salient feature of the limiting distributions obtained (after normalizing in a family-specific manner) is that they only depend on $\alpha$.

In the one-sided variant, the case $t_{n} \equiv 1$ (i.e., $\alpha=0$ ) corresponds to the number of cuts required to disconnect the tree. For this random variable, Meir and

Date: August 4, 2005.

2000 Mathematics Subject Classification. Primary: 68W40; Secondary: 60F05, 60C05.

Key words and phrases. Cutting, Hadamard products, limit laws, method of moments, random spanning tree model, simply generated trees, singularity analysis, Union-Find.

The research of James Allen Fill was supported by NSF Grants DMS-0104167 and DMS0406104, and by The Johns Hopkins University's Acheson J. Duncan Fund for the Advancement of Research in Statistics. Nevin Kapur's research was supported by NSF grant 0049092 and the Center for Mathematics of Information at the California Institute of Technology. 
Moon [13] derived the mean and variance for Cayley trees; Chassaing and Marchand 2] derived the limiting distribution for Cayley trees. Panholzer obtained limiting distributions for non-crossing trees [16] and very simple families of trees [15]. Recently Janson extended these results to all simply generated families [10].

The interest in the two-sided variant stems from the fact that when the very simple family is Cayley trees, the process is equivalent to a probabilistic model (the "random spanning tree model") involved in the Union-Find (or equivalencefinding) algorithm. Knuth and Schönhage 12 derived the expected value of the cost in the cases (among others) $t_{n} \sim a \sqrt{n}$ and $t_{n}=n / 2$. These results were later extended [11] to the cases $t_{n}=n^{\alpha}$ when $\alpha>1 / 2$ and $t_{n}=O\left(n^{\alpha}\right)$ when $\alpha<1 / 2$. (Some of these expected values were rederived using singularity analysis in 4.) In [3], Chassaing and Marchand derive limit laws for the costs considered by Knuth and Schönhage.

We treat both variants of the destruction process using singularity analysis [], a complex-analytic technique that relates asymptotics of sequences to singularities of their generating functions. We rely on applicability of singularity analysis to the Hadamard product (the term-by-term product) of sequences [4] and the amenability of the generalized polylogarithm to singularity analysis 7 .

The organization of the paper is as follows. In Section 2 we define families of very simple trees, noting the key "randomness-preservation" property that is crucial for the application of our methods. Section 3 establishes notation and other preliminaries that will be used in the subsequent proofs. In Section 4 the two-sided variant is considered, and Section 5 deals with the one-sided variant.

Notation. In the sequel we will use ln to denote natural logarithms and log when the base of the logarithm does not matter.

\section{VERY SIMPLE TREES}

An ordered tree is a rooted tree in which the order of the subtrees of each given node is relevant. Given a sequence $\left(\phi_{i}\right)_{i \geq 0}$ of nonnegative numbers (called a degree generating sequence) with $\phi_{0}=1$, a simply generated family $\mathcal{F}$ of trees is obtained by assigning each ordered tree $T$ the weight

$$
w(T):=\prod_{v \in T} \phi_{d(v)},
$$

where $d(v)$ is the outdegree of the node $v$. Let $\mathcal{F}_{n}$ denote the set of trees in $\mathcal{F}$ with $n$ nodes, and let $T_{n}$ denote the weighted number of trees in $\mathcal{F}_{n}$, i.e.,

$$
T_{n}:=\sum_{T \in \mathcal{F}_{n}} w(T) .
$$

A random simply generated tree of size $n$ is obtained by assigning probability $w(T) / T_{n}$ to the tree $T \in \mathcal{F}_{n}$. Many combinatorially interesting families such as (unweighted) ordered trees, Cayley trees, Motzkin trees, and $d$-ary trees are simply generated. It is also well known that simply generated trees correspond to certain conditioned Galton-Watson trees; see the introductory section of [10] for the precise connection. It is well-known that the generating function $T(z):=\sum_{n \geq 1} T_{n} z^{n}$ satisfies the functional equation

$$
T(z)=z \Phi(T(z)),
$$


where $\Phi(t):=\sum_{k \geq 0} \phi_{k} t^{k}$ is the degree generating function of the family. For further background on simply generated trees we refer the reader to [14].

In this paper we consider the subclass of simply generated families, called very simple families, that, among simply generated families, are characterized by the following property.

Choose a random simply generated tree from the family $\mathcal{F}_{n}$ and then one of its $n-1$ edges uniformly at random. Cutting this edge produces a pair of trees of size $k$ (the one that contains the root) and $n-k$, as described in Section 11 Then the subtrees themselves are random simply generated trees from the family $\mathcal{F}_{k}$ and $\mathcal{F}_{n-k}$.

It is clear that the "randomness-preservation" property of very simple trees allows a simple recursive formulation [see (3.1) and [3.12)] of the total cost of destroying such a tree.

Panholzer [15] Lemma 1] characterized the degree generating functions of very simple trees; the relevant constraints are summarized in Table 1

2.1. Singular expansions. As is usual for treatment of simply generated families, let $\tau$ denote the unique root of $t \Phi^{\prime}(t)=\Phi(t)$ with $0<t<R$, where $R$ is the radius of convergence of the series $\Phi$. Let $\rho:=\tau / \Phi(\tau)$. Let $Z:=1-\rho^{-1} z$, and let $\mathcal{A}$ denote a generic power series in $Z$, possibly different at each occurrence. Then as $z \rightarrow \rho$, the dominant singularity for $T(z)$, a singular expansion for $T$ is $[9$, Theorem VII.2]

$$
T(z) \sim \tau-b \rho^{1 / 2} Z^{1 / 2}+Z \mathcal{A}+Z^{3 / 2} \mathcal{A},
$$

where $b:=\Phi(\tau) \sqrt{\frac{2}{\tau \Phi^{\prime \prime}(\tau)}}$. immediately from singularity analysis that

$$
T_{n} \sim c \rho^{-n} n^{-3 / 2}\left(1+n^{-1} \mathcal{N}\right),
$$

where $c:=b \rho^{1 / 2} /(2 \sqrt{\pi})=\left[\Phi(\tau) / 2 \pi \Phi^{\prime \prime}(\tau)\right]^{1 / 2}$. In the sequel we will also use

$$
\sigma^{2}:=\tau^{2} \frac{\Phi^{\prime \prime}(\tau)}{\Phi(\tau)}
$$

Differentiating the expansion (2.1) term-by-term [4, Theorem 6] we get

$$
T^{\prime}(z) \sim \frac{b}{2} \rho^{-1 / 2} Z^{-1 / 2}+\mathcal{A}+Z^{1 / 2} \mathcal{A} .
$$

Since $z=\rho-\rho Z$,

$$
z T^{\prime}(z) \sim \frac{b}{2} \rho^{1 / 2} Z^{-1 / 2}+\mathcal{A}+Z^{1 / 2} \mathcal{A}
$$

The constants $a_{0}$ and $a_{1}$ described by Table 1 are fundamental constants for our analysis; see especially (3.2). Using (2.1) and (2.4) we get

$$
1+2 a_{0} T(z)+a_{1} z T^{\prime}(z) \sim a_{1} \rho^{1 / 2} \frac{b}{2} Z^{-1 / 2}+\mathcal{A}+Z^{1 / 2} \mathcal{A}
$$

and

$$
1-a_{1} T(z) \sim\left(1-a_{1} \tau\right)+a_{1} b \rho^{1 / 2} Z^{1 / 2}+Z \mathcal{A}+Z^{3 / 2} \mathcal{A} .
$$

It is easily verified that for each very simple family $1-a_{1} \tau=0$ (this fact will be used numerous times in subsequent calculations), so that the constant term vanishes in the singular expansion of $1-a_{1} T(z)$. This leads to

$$
z\left[1-a_{1} T(z)\right] \sim \rho^{3 / 2} a_{1} b Z^{1 / 2}+Z \mathcal{A}+Z^{3 / 2} \mathcal{A}
$$


and consequently

$$
z^{-1}\left[1-a_{1} T(z)\right]^{-1} \sim \rho^{-3 / 2} a_{1}^{-1} b^{-1} Z^{-1 / 2}+\mathcal{A}+Z^{1 / 2} \mathcal{A} .
$$

We will also need

$$
\frac{1}{T(z)} \sim \tau^{-1}+\frac{b \rho^{1 / 2}}{\tau^{2}} Z^{1 / 2}+Z \mathcal{A}+Z^{3 / 2} \mathcal{A}
$$

which follows from (2.1).

\section{Preliminaries}

Throughout, $\stackrel{\mathcal{L}}{=}$ denotes equality in law (or distribution) and $\stackrel{\mathcal{L}}{\rightarrow}$ denotes convergence in law. Recall that the Hadamard product of two power series $f$ and $g$, denoted by $f \odot g$, is the power series defined by

$$
(f \odot g)(z) \equiv f(z) \odot g(z):=\sum_{n} f_{n} g_{n} z^{n},
$$

where

$$
f(z)=\sum_{n} f_{n} z^{n} \quad \text { and } \quad g(z)=\sum_{n} g_{n} z^{n}
$$

3.1. Two-sided destruction. The cost of cutting down a very simple tree of size $n$, call it $X_{n}$, satisfies the distributional recurrence

$$
X_{n} \stackrel{\mathcal{L}}{=} X_{K_{n}}+X_{n-K_{n}}^{*}+t_{n}, \quad n \geq 2 ; \quad X_{1}=t_{1},
$$

where $t_{n}$, for $n \geq 2$, is the toll for cutting an edge from a tree of size $n$. Here $K_{n}$, the (random) size of the tree containing the root, is independent of $\left(X_{j}\right)_{j \geq 1}$ and $\left(X_{j}^{*}\right)_{j \geq 1}$, which are independent copies of each other. The splitting probabilities are given by

$$
\operatorname{Pr}\left[K_{n}=k\right]=: p_{n, k}=\left(a_{1} k+a_{0}\right) \frac{T_{k} T_{n-k}}{(n-1) T_{n}}, \quad k=1, \ldots, n-1 .
$$

Table 1 gives the constants $a_{1}$ and $a_{0}$ for each type of very simple family; see (14)(16) in [15]. Here $\alpha_{i}:=\phi_{i+1} / \phi_{i}, i=0,1$, where $\left(\phi_{i}\right)_{i \geq 0}$ is the degree generating sequence of the simply generated tree. It is easy to check that family $\mathrm{A}$ is Cayley

\begin{tabular}{ccccc} 
Family & Generating function & Constraints & $a_{1}$ & $a_{0}$ \\
\hline $\mathrm{A}$ & $e^{\alpha_{0} t}$ & & $\alpha_{0}$ & 0 \\
$\mathrm{~B}$ & $\left(1+\frac{\alpha_{0} t}{d}\right)^{d}$ & $d \geq 2$ & $\alpha_{0} \frac{d-1}{d}$ & $\frac{\alpha_{0}}{d}$ \\
$\mathrm{C}$ & {$\left[1-\left(2 \alpha_{1}-\alpha_{0}\right) t\right]^{-\frac{\alpha_{0}}{2 \alpha_{1}-\alpha_{0}}}$} & $2 \alpha_{1}-\alpha_{0}>0$ & $2 \alpha_{1}$ & $-\left(2 \alpha_{1}-\alpha_{0}\right)$
\end{tabular}

TABLE 1. Generating functions for very simple families. For each family, $\alpha_{0}>0$ is also a constraint.

trees, family $\mathrm{B}$ is $d$-ary trees, and family $\mathrm{C}$ contains unweighted ordered trees. (As it turns out, the distributional recurrence for Cayley trees is identical to the one obtained for the Union-Find process studied in [12, 11, 4] see Remark 4.3 below.) 
Define $\mu_{n}^{[s]}:=\mathbf{E} X_{n}^{s}$. Taking $s$ th powers of both sides of (3.1) and taking expectations by conditioning on $K_{n}$, we get

$$
\mu_{n}^{[s]}=\sum_{k=1}^{n-1} p_{n, k}\left(\mu_{k}^{[s]}+\mu_{n-k}^{[s]}\right)+r_{n}^{[s]}, \quad n \geq 2,
$$

where

$$
r_{n}^{[s]}:=\sum_{\substack{s_{1}+s_{2}+s_{3}=s \\
s_{2}, s_{3}<s}}\left(\begin{array}{c}
s \\
s_{1}, s_{2}, s_{3}
\end{array}\right) t_{n}^{s_{1}} \sum_{k=1}^{n-1} p_{n, k} \mu_{k}^{\left[s_{2}\right]} \mu_{n-k}^{\left[s_{3}\right]},
$$

and $\mu_{1}^{[s]}=t_{1}^{s}$. Define generating functions

$$
\mu^{[s]}(z):=\sum_{n \geq 1} \mu_{n}^{[s]} T_{n} z^{n}, \quad t(z):=\sum_{n \geq 1} t_{n} z^{n}, \quad T(z):=\sum_{n \geq 1} T_{n} z^{n} .
$$

[Observe that $\mu^{[0]}(z)=T(z)$.] Multiply (3.3) by $(n-1) T_{n} z^{n}$ and sum over $n \geq 2$. The resulting left side is

$$
\sum_{n \geq 2}(n-1) T_{n} \mu_{n}^{[s]} z^{n}=\sum_{n \geq 1}(n-1) T_{n} \mu_{n}^{[s]} z^{n}=z \partial_{z} \mu^{[s]}(z)-\mu^{[s]}(z),
$$

where $\partial_{z}$ denotes derivative with respect to $z$. Similarly, the resulting first term on the right side is

$$
a_{1}\left[z\left(\partial_{z} \mu^{[s]}(z)\right) T(z)+z T^{\prime}(z) \mu^{[s]}(z)\right]+2 a_{0} \mu^{[s]}(z) T(z) .
$$

The resulting second term on the right side is

$$
\begin{aligned}
& r^{[s]}(z):=\sum_{n \geq 2}(n-1) T_{n} r_{n}^{[s]} z^{n}=\sum_{n \geq 1}(n-1) T_{n} r_{n}^{[s]} z^{n} \\
& (3.5) \\
& =\sum_{\substack{s_{1}+s_{2}+s_{3}=s \\
s_{2}, s_{3}<s}}\left(\begin{array}{c}
s \\
s_{1}, s_{2}, s_{3}
\end{array}\right) t^{\odot s_{1}}(z) \odot\left[a_{1} z\left(\partial_{z} \mu^{\left[s_{2}\right]}(z)\right) \mu^{\left[s_{3}\right]}(z)+a_{0} \mu^{\left[s_{2}\right]}(z) \mu^{\left[s_{3}\right]}(z)\right] .
\end{aligned}
$$

Thus (3.3) translates to

$$
\begin{aligned}
z \partial_{z} \mu^{[s]}(z) & -\mu^{[s]}(z) \\
& =a_{1}\left[z\left(\partial_{z} \mu^{[s]}(z)\right) T(z)+z T^{\prime}(z) \mu^{[s]}(z)\right]+2 a_{0} \mu^{[s]}(z) T(z)+r^{[s]}(z),
\end{aligned}
$$

i.e.,

$$
\partial_{z} \mu^{[s]}(z)+p(z) \mu^{[s]}(z)=g^{[s]}(z),
$$

where

$$
p(z):=-\frac{1+2 a_{0} T(z)+a_{1} z T^{\prime}(z)}{z\left[1-a_{1} T(z)\right]}
$$

and

$$
g^{[s]}(z):=\frac{r^{[s]}(z)}{z\left[1-a_{1} T(z)\right]},
$$


with $\mu^{[s]}(0)=0$. By variation of parameters (see, for example, 11, 2.1-(22) and Problem 2.1.21], the general solution to the first-order linear differential equation (3.6) is given by

$$
\mu^{[s]}(z)=A^{[s]}(z) \exp \left[-\int_{z_{0}}^{z} p(t) d t\right]
$$

where

$$
A^{[s]}(z):=\int_{0}^{z} g^{[s]}(t) \exp \left[\int_{z_{0}}^{t} p(u) d u\right] d t+\beta_{s}
$$

with $z_{0}$ chosen as follows and $\beta_{s}$ an arbitrary constant.

The integrand $p(z)$ defined at (3.7) and appearing in (3.9) -3.10 is asymptotic to $-1 / z$ as $z \rightarrow 0$ and has [see (4.1) below] another singularity at $z=\rho$. In (3.9) - (3.10) we may choose (and fix) $z_{0}$ arbitrarily from the punctured disc of radius $\rho$ centered at the origin. Then, in (3.10), as $t \rightarrow 0$ we have

$$
\begin{aligned}
\exp \left[\int_{z_{0}}^{t} p(u) d t\right] & =\exp \left[\int_{z_{0}}^{t}\left(-\frac{1}{u}\right) d u+\int_{z_{0}}^{t}\left[p(u)+\frac{1}{u}\right] d u\right] \\
& =\exp \left[-\ln t+\ln z_{0}+\int_{z_{0}}^{t}\left[p(u)+\frac{1}{u}\right] d u\right] \\
& \sim z_{0} e^{a} t^{-1}, \text { where } a:=\int_{z_{0}}^{0}\left[p(u)+\frac{1}{u}\right] d u
\end{aligned}
$$

whereas, using (3.8) and (3.5),

$$
g^{[s]}(t) \sim \frac{r^{[s]}(t)}{t} \sim T_{2} r_{2}^{[s]} t
$$

thus the integrand in (3.10) has no singularity at $t=0$.

Now we obtain the particular solution of interest, using the boundary condition $\mu^{[s]}(z) \sim t_{1}^{s} T_{1} z$ as $z \rightarrow 0$. We find the constant $\beta_{s}$ is specified in terms of $z_{0}$ as

$$
\beta_{s}=z_{0} e^{a} t_{1}^{s} T_{1}
$$

Remark 3.1. One can check for each very simple family that

$$
\Phi^{\prime}(t)=\frac{a_{0}+a_{1}}{1+a_{0} t} \Phi(t)
$$

and for any simply generated family that

$$
T^{\prime}(z)=\frac{\Phi(T(z))}{1-\frac{\Phi^{\prime}(T(z)) T(z)}{\Phi(T(z))}}
$$

Thus

$$
p(z)=-\frac{\Phi(T(z))}{T(z)\left[1-a_{1} T(z)\right]}\left[1+2 a_{0} T(z)+\frac{a_{1} T(z)}{1-\frac{\left(a_{0}+a_{1}\right) T(z)}{1+a_{0} T(z)}}\right]
$$


This leads to

$$
\begin{aligned}
\int_{z_{0}}^{z} p(t) d t & =-\int_{T\left(z_{0}\right)}^{T(z)} \frac{1}{T\left(1-a_{1} T\right)}\left[1+2 a_{0} T+\frac{a_{1} T}{1-\frac{\left(a_{0}+a_{1}\right) T}{1+a_{0} T}}\right]\left[1-\frac{\left(a_{0}+a_{1}\right) T}{1+a_{0} T}\right] d T \\
& =-\int_{T\left(z_{0}\right)}^{T(z)}\left(\frac{1}{T}+\frac{a_{0}}{1+a_{0} T}+\frac{a_{1}}{1-a_{1} T}\right) d T \\
& =\ln \left[\frac{1-a_{1} T(z)}{T(z)\left(1+a_{0} T(z)\right)}\right]-\ln \left[\frac{1-a_{1} T\left(z_{0}\right)}{T\left(z_{0}\right)\left(1+a_{0} T\left(z_{0}\right)\right)}\right]
\end{aligned}
$$

and finally, again using the boundary conditions on $\mu^{[s]}(z)$ as $z \rightarrow 0$, to the following explicit form of (3.9):

$$
\mu^{[s]}(z)=\frac{T(z)\left[1+a_{0} T(z)\right]}{1-a_{1} T(z)}\left\{\int_{0}^{z} g^{[s]}(t) \frac{1-a_{1} T(t)}{T(t)\left[1+a_{0} T(t)\right]} d t+t_{1}^{s}\right\} .
$$

3.2. One-sided destruction. Here, the cost of cutting down a very simple tree of size $n$, call it $Y_{n}$, satisfies the distributional recurrence

$$
Y_{n} \stackrel{\mathcal{L}}{=} Y_{K_{n}}+t_{n}, \quad n \geq 2 ; \quad Y_{1}=t_{1},
$$

where $t_{n}$, for $n \geq 2$, is the toll for cutting an edge from a tree of size $n$ and the splitting probabilities are given by $p_{n, k}$ at (3.2).

Defining $\mu_{n}^{[s]}:=\mathbf{E} Y_{n}^{s}$, one obtains from equation (3.12) by conditioning on $K_{n}$ the recurrence relation

$$
\mu_{n}^{[s]}=\sum_{k=1}^{n-1} p_{n, k} \mu_{k}^{[s]}+r_{n}^{[s]}, \quad n \geq 2
$$

where

$$
r_{n}^{[s]}:=\sum_{\substack{s_{1}+s_{2}=s, s_{2}<s}}\left(\begin{array}{c}
s \\
s_{1}
\end{array}\right) t_{n}^{s_{1}} \sum_{k=1}^{n-1} p_{n, k} \mu_{k}^{\left[s_{2}\right]},
$$

and $\mu_{1}^{[s]}=t_{1}^{s}$. Using the same notation as in Section 3.1] we obtain the following differential equation by multiplying (3.13) by $(n-1) T_{n} z^{n}$ and summing over $n \geq 2$ :

$$
z \partial_{z} \mu^{[s]}(z)-\mu^{[s]}(z)=T(z)\left(a_{1} z \partial_{z} \mu^{[s]}(z)+a_{0} \mu^{[s]}(z)\right)+r^{[s]}(z)
$$

where

$$
r^{[s]}(z):=\sum_{\substack{s_{1}+s_{2}=s, s_{2}<s}}\left(\begin{array}{c}
s \\
s_{1}
\end{array}\right) t^{\odot s_{1}}(z) \odot\left[T(z)\left(a_{1} z \partial_{z} \mu^{\left[s_{2}\right]}(z)+a_{0} \mu^{\left[s_{2}\right]}(z)\right)\right] .
$$

This can be written as

$$
\partial_{z} \mu^{[s]}(z)+p(z) \mu^{[s]}(z)=g^{[s]}(z)
$$

with

$$
p(z):=-\frac{1+a_{0} T(z)}{z\left[1-a_{1} T(z)\right]} \quad \text { and } \quad g^{[s]}(z):=\frac{r^{[s]}(z)}{z\left[1-a_{1} T(z)\right]} .
$$

One can check that for each very simple family, $p(z)=-\partial_{z} \ln (T(z))$, so that we obtain as general solution of the first order linear differential equation (3.15):

$$
\mu^{[s]}(z)=T(z) \int_{0}^{z} \frac{g^{[s]}(t)}{T(t)} d t+C T(z),
$$


and finally by adapting to the initial condition $\left.\partial_{z} \mu^{[s]}(z)\right|_{z=0}=T_{1} \mu_{1}^{[s]}=T_{1} t_{1}^{s}$, that the integration constant is given as $C=t_{1}^{s}$. Therefore, we get

$$
\mu^{[s]}(z)=T(z) \int_{0}^{z} \frac{g^{[s]}(t)}{T(t)} d t+t_{1}^{s} T(z) .
$$

\section{TWO-SIDED DESTRUCTION}

We begin by obtaining a singular expansion for $p(z)$ at (3.7). Using (2.5) and (2.6) in (3.7) we get

$$
p(z) \sim-\frac{\rho^{-1}}{2} Z^{-1}+Z^{-1 / 2} \mathcal{A}+\mathcal{A} .
$$

Integrating this singular expansion term-by-term [4 Theorem 7],

$$
\int_{z_{0}}^{z} p(t) d t \sim-\frac{1}{2} \ln Z^{-1}+\mathcal{A}+Z^{1 / 2} \mathcal{A} .
$$

Thus

$$
\exp \left[-\int_{z_{0}}^{z} p(t) d t\right] \sim \xi Z^{-1 / 2}+\mathcal{A}+Z^{1 / 2} \mathcal{A}
$$

where

$$
\xi:=\left(1-\rho^{-1} z_{0}\right)^{1 / 2} \exp \left[-\int_{z_{0}}^{\rho}\left[p(t)+\frac{\rho^{-1}}{2}\left(1-\rho^{-1} t\right)^{-1}\right] d t\right] .
$$

Taking the reciprocal of (4.2) gives

$$
\exp \left[\int_{z_{0}}^{z} p(t) d t\right] \sim \xi^{-1} Z^{1 / 2}+Z \mathcal{A}+Z^{3 / 2} \mathcal{A} .
$$

Let us now consider two-sided destruction with the toll $t_{n}=n^{\alpha}$, with $\alpha>0$. (Notice that the case $\alpha=0$ is trivial since then the total cost of destruction is simply the number of edges in the tree, which is always $n-1$.) The toll generating function $t(z)$ is the generalized polylogarithm $\mathrm{Li}_{-\alpha, 0}(z)$, which is amenable to singularity analysis [7] Theorem 1].

4.1. Expectation. Now we obtain a singular expansion for $r^{[1]}(z)$ defined at (3.5), recalling that $\mu^{[0]}(z)=T(z)$ :

$$
r^{[1]}(z)=t(z) \odot\left[a_{1} z T^{\prime}(z) T(z)+a_{0} T^{2}(z)\right] .
$$

Using (2.1) we conclude that

$$
T^{2}(z) \sim \mathcal{A}+Z^{1 / 2} \mathcal{A}
$$

and using (2.4) that

$$
a_{1} z T^{\prime}(z) T(z)+a_{0} T^{2}(z) \sim \rho^{1 / 2} \frac{b}{2} Z^{-1 / 2}+\mathcal{A}+Z^{1 / 2} \mathcal{A} .
$$

We will use the Zigzag algorithm of 4 to obtain a singular expansion for $r^{[1]}(z)$. We recall the use of the notation $\mathcal{N}$ to denote a generic power series in $1 / n$, possibly different at each occurrence. By singularity analysis,

$$
\rho^{n}\left[z^{n}\right]\left[a_{1} z T^{\prime}(z) T(z)+a_{0} T^{2}(z)\right] \sim \rho^{1 / 2} \frac{b}{2} \frac{n^{-1 / 2}}{\sqrt{\pi}}+n^{-3 / 2} \mathcal{N} .
$$


Thus

$$
\rho^{n}\left[z^{n}\right] r^{[1]}(z) \sim \rho^{1 / 2} \frac{b}{2} \frac{n^{\alpha-\frac{1}{2}}}{\sqrt{\pi}}+n^{\alpha-\frac{3}{2}} \mathcal{N} .
$$

Until further notice, suppose $\alpha \notin\left\{\frac{1}{2}, \frac{3}{2}, \ldots\right\}$. Then a compatible singular expansion for $r^{[1]}(z)$ at (4.5) is obtained as

$$
r^{[1]}(z) \sim \rho^{1 / 2} b \frac{\Gamma\left(\alpha+\frac{1}{2}\right)}{2 \sqrt{\pi}} Z^{-\alpha-\frac{1}{2}}+Z^{-\alpha+\frac{1}{2}} \mathcal{A}+\mathcal{A} .
$$

Recalling (3.8) and (2.6) we have

$$
g^{[1]}(z) \sim \frac{\tau}{\rho} \frac{\Gamma\left(\alpha+\frac{1}{2}\right)}{2 \sqrt{\pi}} Z^{-\alpha-1}+Z^{-\alpha-\frac{1}{2}} \mathcal{A}+Z^{-\alpha} \mathcal{A}+Z^{-1 / 2} \mathcal{A}+\mathcal{A}
$$

Using this expansion and (4.4),

$$
g^{[1]}(z) \exp \left[\int_{z_{0}}^{z} p(t) d t\right] \sim \frac{\tau}{\xi \rho} \frac{\Gamma\left(\alpha+\frac{1}{2}\right)}{2 \sqrt{\pi}} Z^{-\alpha-\frac{1}{2}}+Z^{-\alpha} \mathcal{A}+Z^{-\alpha+\frac{1}{2}} \mathcal{A}+\mathcal{A}+Z^{1 / 2} \mathcal{A} .
$$

By (3.10) and Theorem 7 of [4, we may integrate this expansion term-by-term to get a complete singular expansion for $A$. If $\alpha \notin\{1,2, \ldots\}$, we have

$$
A^{[1]}(z) \sim \frac{\tau}{\xi} \frac{\Gamma\left(\alpha-\frac{1}{2}\right)}{2 \sqrt{\pi}} Z^{-\alpha+\frac{1}{2}}+L_{0}+Z^{-\alpha+1} \mathcal{A}+Z^{-\alpha+\frac{3}{2}} \mathcal{A}+Z \mathcal{A}+Z^{3 / 2} \mathcal{A}
$$

where $L_{0}$ is a constant. [The value of $L_{0}$ is immaterial unless $0<\alpha<1 / 2$, in which case see (4.14).] On the other hand, if $\alpha \in\{1,2, \ldots\}$, a logarithmic term appears upon integration, so that

$$
A^{[1]}(z) \sim \frac{\tau}{\xi} \frac{\Gamma\left(\alpha-\frac{1}{2}\right)}{2 \sqrt{\pi}} Z^{-\alpha+\frac{1}{2}}+Z^{-\alpha+1} \mathcal{A}+Z^{-\alpha+\frac{3}{2}} \mathcal{A}+K_{0} \ln Z^{-1},
$$

where $K_{0}$ is a constant. Combining these expansions with (4.2), we finally obtain [recalling (3.9)]

$$
\mu^{[1]}(z) \sim \tau \frac{\Gamma\left(\alpha-\frac{1}{2}\right)}{2 \sqrt{\pi}} Z^{-\alpha}+L_{0} \xi Z^{-1 / 2}+Z^{-\alpha+\frac{1}{2}} \mathcal{A}+Z^{-\alpha+1} \mathcal{A}+\mathcal{A}+Z^{1 / 2} \mathcal{A}
$$

when $\alpha \notin\{1,2, \ldots\}$ and

$$
\mu^{[1]}(z) \sim \tau \frac{\Gamma\left(\alpha-\frac{1}{2}\right)}{2 \sqrt{\pi}} Z^{-\alpha}+Z^{-\alpha+\frac{1}{2}} \mathcal{A}+Z^{-\alpha+1} \mathcal{A}+Z^{-1 / 2}\left(\ln Z^{-1}\right) \mathcal{A}+\left(\ln Z^{-1}\right) \mathcal{A}
$$

when $\alpha \in\{1,2, \ldots\}$. Note that the remainder in (4.12) is $O\left(|Z|^{-\alpha+\frac{1}{2}}\right)$ unless $\alpha=1$, in which case it is $O\left(|Z|^{-\frac{1}{2}} \ln Z^{-1}\right)=O\left(|Z|^{-\frac{1}{2}-\epsilon}\right)$ for any $\epsilon>0$.

When $\alpha>1 / 2$ and $\alpha \notin\{1,2, \ldots\}$, by singularity analysis we have

$$
\rho^{n} \mu_{n}^{[1]} T_{n} \sim \tau \frac{\Gamma\left(\alpha-\frac{1}{2}\right)}{2 \sqrt{\pi} \Gamma(\alpha)} n^{\alpha-1}+n^{\alpha-\frac{3}{2}} \mathcal{N}+n^{\alpha-2} \mathcal{N}+n^{-1 / 2} \mathcal{N}
$$

so that, recalling (2.2) and (2.3),

$$
\mu_{n}^{[1]} \sim \sigma \frac{\Gamma\left(\alpha-\frac{1}{2}\right)}{\sqrt{2} \Gamma(\alpha)} n^{\alpha+\frac{1}{2}}+n^{\alpha} \mathcal{N}+n^{\alpha-\frac{1}{2}} \mathcal{N}+n \mathcal{N}
$$


When $\alpha \in\{1,2, \ldots\}$, starting from (4.12) and the note following that display, we can similarly derive the expansion

$$
\mu_{n}^{[1]}=\sigma \frac{\Gamma\left(\alpha-\frac{1}{2}\right)}{\sqrt{2} \Gamma(\alpha)} n^{\alpha+\frac{1}{2}}+O\left(n^{\alpha}\right)+O(n \log n) .
$$

When $0<\alpha<1 / 2$, a similar computation yields

$$
\mu_{n}^{[1]} \sim \frac{L_{0} \xi}{c \sqrt{\pi}} n+\sigma \frac{\Gamma\left(\alpha-\frac{1}{2}\right)}{\sqrt{2} \Gamma(\alpha)} n^{\alpha+\frac{1}{2}}+n^{\alpha} \mathcal{N}+n^{\alpha-\frac{1}{2}} \mathcal{N}+\mathcal{N}
$$

where

$$
L_{0}:=\int_{0}^{\rho} g^{[1]}(t) \exp \left[\int_{z_{0}}^{t} p(u) d u\right] d t+\beta_{1}
$$

with $p$ and $g^{[1]}$ defined at (3.77) and (3.8), respectively, and $\xi$ and $\beta_{1}$ at (4.3) and (3.11), respectively.

When $\alpha \in\left\{\frac{3}{2}, \frac{5}{2}, \ldots\right\}$, one can check that logarithmic terms appear in the singular expansion compatible with (4.8) but the lead-order term and asymptotic order of the remainder are unchanged. Indeed, now

$$
\mu^{[1]}(z)=\tau \frac{\Gamma\left(\alpha-\frac{1}{2}\right)}{2 \sqrt{\pi}} Z^{-\alpha}+O\left(|Z|^{-\alpha+\frac{1}{2}}\right)
$$

and consequently

$$
\mu_{n}^{[1]}=\sigma \frac{\Gamma\left(\alpha-\frac{1}{2}\right)}{\sqrt{2} \Gamma(\alpha)} n^{\alpha+\frac{1}{2}}+O\left(n^{\alpha}\right) .
$$

Finally we consider $\alpha=1 / 2$. Now, a compatible singular expansion for (4.8) is

$$
r^{[1]}(z) \sim \frac{\rho^{1 / 2} b}{2 \sqrt{\pi}} Z^{-1}+(\log Z) \mathcal{A}+\mathcal{A}
$$

Proceeding as in the case $\alpha \neq 1 / 2$ we have the singular expansions

$$
\begin{array}{r}
g^{[1]}(z) \sim \frac{\tau}{2 \rho \sqrt{\pi}} Z^{-3 / 2}+Z^{-1} \mathcal{A}+\left(Z^{-1 / 2} \log Z\right) \mathcal{A}+Z^{-1 / 2} \mathcal{A}+(\log Z) \mathcal{A}, \\
g^{[1]}(z) \exp \left[\int_{z_{0}}^{z} p(t) d t\right] \sim \frac{\tau}{\xi \rho} \frac{1}{2 \sqrt{\pi}} Z^{-1}+Z^{-\frac{1}{2}} \mathcal{A}+(\log Z) \mathcal{A}+\mathcal{A}+\left(Z^{1 / 2} \log Z\right) \mathcal{A}, \\
A^{[1]}(z) \sim \frac{\tau}{\xi} \frac{1}{2 \sqrt{\pi}} \ln Z^{-1}+L_{1}+Z^{1 / 2} \mathcal{A}+Z \mathcal{A}+(Z \log Z) \mathcal{A}+\left(Z^{3 / 2} \log Z\right) \mathcal{A},
\end{array}
$$

where

$$
L_{1}:=\int_{0}^{\rho}\left\{g^{[1]}(t) \exp \left[\int_{z_{0}}^{t} p(u) d u\right]-\frac{\tau}{\xi \rho} \frac{1}{2 \sqrt{\pi}}\left(1-\rho^{-1} t\right)\right\} d t+\beta_{1} .
$$

This leads to

$\mu^{[1]}(z) \sim \frac{\tau}{2 \sqrt{\pi}} Z^{-1 / 2} \ln Z^{-1}+\xi L_{1} Z^{-1 / 2}+(\log Z) \mathcal{A}+\mathcal{A}+\left(Z^{1 / 2} \log Z\right) \mathcal{A}+Z^{1 / 2} \mathcal{A}$,

so that by singularity analysis and (2.2) we have

$$
\begin{array}{r}
\mu_{n}^{[1]} \sim \frac{\sigma}{\sqrt{2 \pi}} n \ln n+\left[\frac{\xi L_{1}}{c \sqrt{\pi}}+\frac{\sigma}{\sqrt{2 \pi}}(\gamma+2 \ln 2)\right] n \\
+\left(n^{1 / 2} \log n\right) \mathcal{N}+(\log n) \mathcal{N}+\mathcal{N},
\end{array}
$$

where $\sigma$ is defined at (2.3). 
4.2. Higher moments and limiting distributions. We proceed to higher moments. We will consider separately the cases $\alpha>1 / 2, \alpha<1 / 2$, and $\alpha=1 / 2$. We present the details for $\alpha>1 / 2$ and sketch the main ideas for the other cases. Throughout $\alpha^{\prime}:=\alpha+\frac{1}{2}$.

Proposition 4.1. Let $\alpha>1 / 2$ and $\epsilon>0$. Then

$$
\mu^{[s]}(z)=C_{s} Z^{-s \alpha^{\prime}+\frac{1}{2}}+O\left(|Z|^{-s \alpha^{\prime}+\frac{1}{2}+q}\right),
$$

where

$$
q:= \begin{cases}\min \left\{\alpha-\frac{1}{2}, \frac{1}{2}\right\} & \text { if } \alpha \neq 1 \\ \frac{1}{2}-\epsilon & \text { if } \alpha=1\end{cases}
$$

with

$$
C_{1}=\tau \frac{\Gamma\left(\alpha-\frac{1}{2}\right)}{2 \sqrt{\pi}}
$$

and, for $s \geq 2$,

$C_{s}=\rho^{-1 / 2} b^{-1}\left[\frac{1}{s \alpha^{\prime}-1} \sum_{k=1}^{s-1}\left(\begin{array}{l}s \\ k\end{array}\right)\left(k \alpha^{\prime}-\frac{1}{2}\right) C_{k} C_{s-k}+s \tau \frac{\Gamma\left(s \alpha^{\prime}-1\right)}{\Gamma\left((s-1) \alpha^{\prime}-\frac{1}{2}\right)} C_{s-1}\right]$.

Proof. The proof is by induction on $s$. The claim is true for $s=1$ by (4.11), 4.12), and (4.15). Suppose $s \geq 2$. We analyze each term in the sum for $r^{[s]}(z)$ at (3.5).

If both $s_{2}$ and $s_{3}$ are nonzero, then by the induction hypothesis,

$$
z \partial_{z} \mu^{\left[s_{2}\right]}(z)=C_{s_{2}}\left(s_{2} \alpha^{\prime}-\frac{1}{2}\right) Z^{-s_{2} \alpha^{\prime}-\frac{1}{2}}+O\left(|Z|^{-s_{2} \alpha^{\prime}-\frac{1}{2}+q}\right),
$$

so that

$$
z\left(\partial_{z} \mu^{\left[s_{2}\right]}(z)\right) \mu^{\left[s_{3}\right]}(z)=C_{s_{2}} C_{s_{3}}\left(s_{2} \alpha^{\prime}-\frac{1}{2}\right) Z^{-\left(s_{2}+s_{3}\right) \alpha^{\prime}}+O\left(|Z|^{-\left(s_{2}+s_{3}\right) \alpha^{\prime}+q}\right) .
$$

Also, $\mu^{\left[s_{2}\right]}(z) \mu^{\left[s_{3}\right]}(z)=O\left(|Z|^{-\left(s_{2}+s_{3}\right) \alpha^{\prime}+1}\right)$. Hence

$$
\begin{aligned}
a_{1} z\left(\partial_{z} \mu^{\left[s_{2}\right]}(z)\right) \mu^{\left[s_{3}\right]}(z)+a_{0} \mu^{\left[s_{2}\right]}(z) \mu^{\left[s_{3}\right]}(z) & \\
& =a_{1} C_{s_{2}} C_{s_{3}}\left(s_{2} \alpha^{\prime}-\frac{1}{2}\right) Z^{-\left(s_{2}+s_{3}\right) \alpha^{\prime}}+O\left(|Z|^{-\left(s_{2}+s_{3}\right) \alpha^{\prime}+q}\right) .
\end{aligned}
$$

Taking the Hadamard product of this expansion with $t^{\odot s_{1}}(z)$ (using the Zigzag algorithm again) gives the contribution of such terms to $r^{[s]}(z)$ as

$$
\left(\begin{array}{c}
s \\
s_{1}, s_{2}, s_{3}
\end{array}\right) a_{1} C_{s_{2}} C_{s_{3}}\left(s_{2} \alpha^{\prime}-\frac{1}{2}\right) \frac{\Gamma\left(s \alpha^{\prime}-\frac{s_{1}}{2}\right)}{\Gamma\left(\left(s_{2}+s_{3}\right) \alpha^{\prime}\right)} Z^{-\left(s \alpha^{\prime}-\frac{s_{1}}{2}\right)}+O\left(|Z|^{-\left(s \alpha^{\prime}-\frac{s_{1}}{2}\right)+q}\right) .
$$

Notice that if $s_{1} \neq 0$ the contribution is $O\left(|Z|^{-s \alpha^{\prime}+\frac{1}{2}}\right)$.

Next consider the case when $s_{2}$ is nonzero but $s_{3}=0$. By the induction hypothesis and the singular expansion of $T$ at (2.1),

$$
z\left(\partial_{z} \mu^{\left[s_{2}\right]}(z)\right) T(z)=\tau C_{s_{2}}\left(s_{2} \alpha^{\prime}-\frac{1}{2}\right) Z^{-s_{2} \alpha^{\prime}-\frac{1}{2}}+O\left(|Z|^{-s_{2} \alpha^{\prime}-\frac{1}{2}+q}\right) .
$$


Also $\mu^{\left[s_{2}\right]}(z) T(z)=O\left(|Z|^{-s_{2} \alpha^{\prime}+\frac{1}{2}}\right)$. Hence

$$
\begin{aligned}
& a_{1} z\left(\partial_{z} \mu^{\left[s_{2}\right]}(z)\right) T(z)+a_{0} \mu^{\left[s_{2}\right]}(z) T(z) \\
& \quad=C_{s_{2}}\left(s_{2} \alpha^{\prime}-\frac{1}{2}\right) Z^{-s_{2} \alpha^{\prime}-\frac{1}{2}}+O\left(|Z|^{-s_{2} \alpha^{\prime}-\frac{1}{2}+q}\right) .
\end{aligned}
$$

Taking the Hadamard product of this singular expansion with $t^{\odot s_{1}}(z)$ we get that the contribution to $r^{[s]}(z)$ from such terms is

$$
\left(\begin{array}{c}
s \\
s_{1}
\end{array}\right) C_{s_{2}}\left(s_{2} \alpha^{\prime}-\frac{1}{2}\right) \frac{\Gamma\left(s \alpha^{\prime}-\frac{s_{1}}{2}+\frac{1}{2}\right)}{\Gamma\left(s_{2} \alpha^{\prime}+\frac{1}{2}\right)} Z^{-\left(s \alpha^{\prime}-\frac{s_{1}}{2}+\frac{1}{2}\right)}+O\left(|Z|^{-\left(s \alpha^{\prime}-\frac{s_{1}}{2}+\frac{1}{2}\right)+q}\right) .
$$

Notice that $s_{1} \geq 1$ and that when $s_{1}>1$ the contribution of such terms is $O\left(|Z|^{-s \alpha^{\prime}+\frac{1}{2}}\right)$.

We move on to the case when $s_{2}=0$ but $s_{3}$ is nonzero. By the induction hypothesis, (2.1), and (2.4), we have $T(z) \mu^{\left[s_{3}\right]}(z)=O\left(|Z|^{-s_{3} \alpha^{\prime}+\frac{1}{2}}\right)$ and $z T^{\prime}(z) \mu^{\left[s_{3}\right]}(z)=$ $O\left(|Z|^{-s_{3} \alpha^{\prime}}\right)$. Thus

$$
a_{1} z T^{\prime}(z) \mu^{\left[s_{3}\right]}(z)+a_{0} T(z) \mu^{\left[s_{3}\right]}(z)=O\left(|Z|^{-s_{3} \alpha^{\prime}}\right) .
$$

Taking the Hadamard product with $t^{\odot s_{1}}(z)$ we see (recalling $s_{1} \geq 1$ ) that the contribution to $r^{[s]}(z)$ from these terms is $O\left(|Z|^{-s \alpha^{\prime}+\frac{1}{2}}\right)$.

Finally we consider the case when $s_{2}=s_{3}=0$. In this case, using (4.6) it is easy to verify that the contribution to $r^{[s]}(z)$ from this term is $O\left(|Z|^{-s \alpha^{\prime}+\frac{1}{2}}\right)$.

Summing all the contributions we see that

$$
r^{[s]}(z)=D_{s} Z^{-s \alpha^{\prime}}+O\left(|Z|^{-s \alpha^{\prime}+q}\right),
$$

where

$$
D_{s}:=a_{1}\left[\sum_{k=1}^{s-1}\left(\begin{array}{l}
s \\
k
\end{array}\right)\left(k \alpha^{\prime}-\frac{1}{2}\right) C_{k} C_{s-k}+s \tau \frac{\left[(s-1) \alpha^{\prime}-\frac{1}{2}\right] \Gamma\left(s \alpha^{\prime}\right)}{\Gamma\left((s-1) \alpha^{\prime}+\frac{1}{2}\right)} C_{s-1}\right]
$$

Thus, using (3.8) and (2.6),

$$
g^{[s]}(z)=\rho^{-3 / 2} a_{1}^{-1} b^{-1} D_{s} Z^{-s \alpha^{\prime}-\frac{1}{2}}+O\left(|Z|^{-s \alpha^{\prime}-\frac{1}{2}+q}\right),
$$

whence, using (4.4),

$$
g^{[s]}(z) \exp \left[\int_{z_{0}}^{z} p(t) d t\right]=\xi^{-1} \rho^{-3 / 2} a_{1}^{-1} b^{-1} D_{s} Z^{-s \alpha^{\prime}}+O\left(|Z|^{-s \alpha^{\prime}+q}\right) .
$$

To get $A^{[s]}(z)$ at (3.10) we integrate this singular expansion, noting that since $s \geq 2$ and $\alpha^{\prime}>1$, we have $s \alpha^{\prime}>2$. Hence

$$
A^{[s]}(z)=\xi^{-1} \rho^{-1 / 2} a_{1}^{-1} b^{-1} \frac{D_{s}}{s \alpha^{\prime}-1} Z^{-s \alpha^{\prime}+1}+O\left(|Z|^{-s \alpha^{\prime}+1+q}\right) .
$$

Now by (3.9) and (4.2),

$$
\mu^{[s]}(z)=\rho^{-1 / 2} a_{1}^{-1} b^{-1} \frac{D_{s}}{s \alpha^{\prime}-1} Z^{-s \alpha^{\prime}+\frac{1}{2}}+O\left(|Z|^{-s \alpha^{\prime}+\frac{1}{2}+q}\right) .
$$


Taking

$$
\begin{aligned}
C_{s} & =\rho^{-1 / 2} a_{1}^{-1} b^{-1} \frac{D_{s}}{s \alpha^{\prime}-1} \\
& =\frac{\rho^{-1 / 2} b^{-1}}{s \alpha^{\prime}-1}\left[\sum_{k=1}^{s-1}\left(\begin{array}{l}
s \\
k
\end{array}\right)\left(k \alpha^{\prime}-\frac{1}{2}\right) C_{k} C_{s-k}+s \tau \frac{\left[(s-1) \alpha^{\prime}-\frac{1}{2}\right] \Gamma\left(s \alpha^{\prime}\right)}{\Gamma\left((s-1) \alpha^{\prime}+\frac{1}{2}\right)} C_{s-1}\right] \\
& =\rho^{-1 / 2} b^{-1}\left[\frac{1}{s \alpha^{\prime}-1} \sum_{k=1}^{s-1}\left(\begin{array}{l}
s \\
k
\end{array}\right)\left(k \alpha^{\prime}-\frac{1}{2}\right) C_{k} C_{s-k}+s \tau \frac{\Gamma\left(s \alpha^{\prime}-1\right)}{\Gamma\left((s-1) \alpha^{\prime}-\frac{1}{2}\right)} C_{s-1}\right]
\end{aligned}
$$

completes the proof.

Using singularity analysis we can now derive asymptotics for the moments $\mu_{n}^{[s]}$.

Theorem 4.2. Let $\alpha>1 / 2$. Then, as $n \rightarrow \infty$,

$$
\sigma^{-s} n^{-s \alpha^{\prime}} \mu_{n}^{[s]} \rightarrow m_{s},
$$

where $\sigma^{2}:=\tau^{2} \frac{\Phi^{\prime \prime}(\tau)}{\Phi(\tau)}$ and $m_{s}$ (which does not depend on the very simple family) is given by

$$
m_{1}=\frac{\Gamma\left(\alpha-\frac{1}{2}\right)}{\sqrt{2} \Gamma(\alpha)}
$$

and, for $s \geq 2$,

$$
m_{s}=\frac{1}{4 \sqrt{\pi}} \sum_{k=1}^{s-1}\left(\begin{array}{l}
s \\
k
\end{array}\right) \frac{\Gamma\left(k \alpha^{\prime}-\frac{1}{2}\right) \Gamma\left((s-k) \alpha^{\prime}-\frac{1}{2}\right)}{\Gamma\left(s \alpha^{\prime}-\frac{1}{2}\right)} m_{k} m_{s-k}+\frac{s \Gamma\left(s \alpha^{\prime}-1\right)}{\sqrt{2} \Gamma\left(s \alpha^{\prime}-\frac{1}{2}\right)} m_{s-1} .
$$

Proof. Using singularity analysis and Proposition 4.1

$$
\rho^{n} \mu_{n}^{[s]} T_{n}=C_{s} \frac{n^{s \alpha^{\prime}-\frac{3}{2}}}{\Gamma\left(s \alpha^{\prime}-\frac{1}{2}\right)}+O\left(n^{s \alpha^{\prime}-\frac{3}{2}-q}\right)
$$

and using the asymptotics of $T_{n}$ at (2.2),

$$
\mu_{n}^{[s]}=\frac{C_{s}}{c \Gamma\left(s \alpha^{\prime}-\frac{1}{2}\right)} n^{s \alpha^{\prime}}+O\left(n^{s \alpha^{\prime}-q}\right) .
$$

Then

$$
\sigma^{-s} n^{-s \alpha^{\prime}} \mu_{n}^{[s]} \rightarrow m_{s}
$$

where

$$
m_{s}:=\sigma^{-s} \frac{C_{s}}{c \Gamma\left(s \alpha^{\prime}-\frac{1}{2}\right)} .
$$

Thus, using $2 \sqrt{\pi} c \sigma=\sqrt{2} \tau$,

$$
m_{1}=\frac{C_{1}}{c \sigma \Gamma(\alpha)}=\frac{\Gamma\left(\alpha-\frac{1}{2}\right)}{\sqrt{2} \Gamma(\alpha)}
$$

Using (4.18), (4.21), and the identities

$$
c \rho^{-1 / 2} b^{-1}=\frac{1}{2 \sqrt{\pi}}, \quad \sigma^{-1} \tau \rho^{-1 / 2} b^{-1}=\frac{1}{\sqrt{2}}, \quad \Gamma(x+1)=x \Gamma(x),
$$


we obtain the following recurrence for $m_{s}$ :

$$
m_{s}=\frac{1}{2 \sqrt{\pi}} \sum_{k=1}^{s-1}\left(\begin{array}{l}
s \\
k
\end{array}\right) \frac{\Gamma\left(k \alpha^{\prime}+\frac{1}{2}\right) \Gamma\left((s-k) \alpha^{\prime}-\frac{1}{2}\right)}{\left(s \alpha^{\prime}-1\right) \Gamma\left(s \alpha^{\prime}-\frac{1}{2}\right)} m_{k} m_{s-k}+\frac{s \Gamma\left(s \alpha^{\prime}-1\right)}{\sqrt{2} \Gamma\left(s \alpha^{\prime}-\frac{1}{2}\right)} m_{s-1} .
$$

To obtain the form of the recurrence in (4.20), write (4.22) in the form

$$
m_{s}=\frac{1}{2 \sqrt{\pi}} \sum_{k=1}^{s-1} e_{s, k}+\tilde{e}_{s}=\frac{1}{4 \sqrt{\pi}} \sum_{k=1}^{s-1}\left(e_{s, k}+e_{s, s-k}\right)+\tilde{e}_{s}
$$

and simplify.

Remark 4.3. In going from (4.22) to (4.20) we symmetrized by collecting coefficients of $m_{k} m_{s-k}$. We might also have symmetrized from the start by choosing the splitting probabilities as

$$
\tilde{p}_{n, k}:=\frac{1}{2}\left(p_{n, k}+p_{n, n-k}\right) .
$$

In the particular case of Cayley trees this leads to the same splitting probabilities as for the Union-Find recurrence studied in [12, 11, 4].

We can now show convergence in distribution via the method of moments.

Theorem 4.4. Let $\alpha>1 / 2$. Define $\sigma^{2}:=\tau^{2} \Phi^{\prime \prime}(\tau) / \Phi(\tau)$ and $\alpha^{\prime}:=\alpha+\frac{1}{2}$. Then, as $n \rightarrow \infty$,

$$
\sigma^{-1} n^{-\alpha^{\prime}} X_{n} \stackrel{\mathcal{L}}{\rightarrow} X^{(\alpha)}
$$

with convergence of all moments, where $X^{(\alpha)}$ has the unique distribution whose sth moment $m_{s} \equiv m_{s}(\alpha)$ is given by

$$
m_{1}=\frac{\Gamma\left(\alpha-\frac{1}{2}\right)}{\sqrt{2} \Gamma(\alpha)}
$$

and for $s \geq 2$ by the recurrence (4.20).

Proof. One need only check that the $m_{k}$ 's satisfy Carleman's condition. This has already been established in [6].

Remark 4.5. It is curious that $\sigma^{-1} n^{-\alpha^{\prime}} X_{n}$ has the same limiting distribution as

$$
\sigma n^{-\alpha^{\prime}} \sum_{v \in T}\left|T_{v}\right|^{\alpha}
$$

Here $T$ is a random simply generated tree and $\left|T_{v}\right|$ denotes the size of the tree rooted at a node $v$. This was established in [5].

For the case $0<\alpha<1 / 2$ it is convenient instead to consider the random variable

$$
\widetilde{X}_{n}:=X_{n}-\mu n, \quad \mu:=\frac{L_{0} \xi}{c \sqrt{\pi}} .
$$

[Note that, by (4.13), $\mu n$ is the lead term in the asymptotics of $\mathbf{E} X_{n}$ when $\alpha<1 / 2$.] Using (3.1),

$$
\widetilde{X}_{n} \stackrel{\mathcal{L}}{=} \widetilde{X}_{K_{n}}+\widetilde{X}_{n-K_{n}}^{*}+t_{n}, \quad n \geq 2 ; \quad \widetilde{X}_{1}=t_{1}-\mu
$$


Define $\tilde{\mu}_{n}^{[s]}:=\mathbf{E} \widetilde{X}_{n}^{s}$ and

$$
\tilde{\mu}^{[s]}(z):=\sum_{n \geq 1} \tilde{\mu}_{n}^{[s]} T_{n} z^{n} .
$$

Then, in analogous fashion, (3.5) - 3.10) hold with $\mu^{[s]}(z)$ replaced by $\tilde{\mu}^{[s]}(z)$.

Observe that, by (4.11),

$$
\tilde{\mu}^{[1]}(z) \sim \tau \frac{\Gamma\left(\alpha-\frac{1}{2}\right)}{2 \sqrt{\pi}} Z^{-\alpha}+\left(Z^{-\alpha+\frac{1}{2}}+Z^{-\alpha+1}+1+Z^{1 / 2}\right) \mathcal{A} .
$$

We can use (4.24) and (3.5) -3.10) to show that Proposition 4.1 holds for $\alpha<1 / 2$ with $\mu^{[s]}(z)$ replaced by $\tilde{\mu}^{[s]}(z)$ and $q$ changed to $2 \alpha-\epsilon$, for sufficiently small $\epsilon>0$. It follows then that $X_{n}-\mu n$ has (after scaling) a limiting distribution.

Theorem 4.6. Let $\alpha<1 / 2$. Define $\sigma^{2}:=\tau^{2} \Phi^{\prime \prime}(\tau) / \Phi(\tau)$ and $\alpha^{\prime}:=\alpha+\frac{1}{2}$. Then, as $n \rightarrow \infty$,

$$
\sigma^{-1} n^{-\alpha^{\prime}}\left(X_{n}-\mu n\right) \stackrel{\mathcal{L}}{\rightarrow} X^{(\alpha)},
$$

with convergence of all moments, where $X^{(\alpha)}$ has the unique distribution whose sth moment $m_{s} \equiv m_{s}(\alpha)$ is given for $s=1$ by

$$
m_{1}=\frac{\Gamma\left(\alpha-\frac{1}{2}\right)}{\sqrt{2} \Gamma(\alpha)}
$$

and for $s \geq 2$ by the recurrence (4.20).

Finally we turn our attention to the case $\alpha=1 / 2$. Now, we define

$$
\widetilde{X}_{n}:=X_{n}-\frac{\sigma}{\sqrt{2 \pi}} n \ln n-\delta n \text { with } \delta:=\frac{\xi L_{1}}{c \sqrt{\pi}}+\frac{\sigma}{\sqrt{2 \pi}}(\gamma+2 \ln 2),
$$

with $L_{1}$ defined at (4.16). Then [cf. (3.1)]

$$
\widetilde{X}_{n} \stackrel{\mathcal{L}}{=} \widetilde{X}_{K_{n}}+\widetilde{X}_{n-K_{n}}^{*}+t_{n, K_{n}}, \quad n \geq 2
$$

with $\widetilde{X}_{1}=1-\delta$ and

$$
t_{n, k}:=\frac{\sigma}{\sqrt{2 \pi}}\left[k \ln k+(n-k) \ln (n-k)-n \ln n+\frac{\sqrt{2 \pi}}{\sigma} n^{1 / 2}\right] .
$$

As in the case $\alpha<1 / 2$, it is easily checked that [3.5 bold with $\mu^{[s]}(z)$ replaced by $\tilde{\mu}^{[s]}(z)$ and $r_{n}^{[s]}$ at (3.4) replaced by

$$
\tilde{r}_{n}^{[s]}:=\sum_{\substack{s_{1}+s_{2}+s_{3}=s \\
s_{2}, s_{3}<s}}\left(\begin{array}{c}
s \\
s_{1}, s_{2}, s_{3}
\end{array}\right) \sum_{k=1}^{n-1} p_{n, k} t_{n, k}^{s_{1}} \tilde{\mu}_{k}^{\left[s_{2}\right]} \tilde{\mu}_{n-k}^{\left[s_{3}\right]} .
$$

The limiting distribution is given by the following result.

Theorem 4.7. As $n \rightarrow \infty$,

$$
\sigma^{-s} n^{-s} \tilde{\mu}_{n}^{[s]} \rightarrow m_{s},
$$

where $m_{0}=1, m_{1}=0$, and for $s \geq 2$,

$$
m_{s}=\frac{\Gamma(s-1)}{2 \sqrt{\pi} \Gamma\left(s-\frac{1}{2}\right)} \sum_{\substack{s_{1}+s_{2}+s_{3}=s \\
s_{2}, s_{3}<s}}\left(\begin{array}{c}
s \\
s_{1}, s_{2}, s_{3}
\end{array}\right)\left(\frac{1}{\sqrt{2 \pi}}\right)^{s_{1}} m_{s_{2}} m_{s_{3}} J_{s_{1}, s_{2}, s_{3}},
$$


with

$$
J_{s_{1}, s_{2}, s_{3}}:=\int_{0}^{1}[x \ln x+(1-x) \ln (1-x)]^{s_{1}} x^{s_{2}-\frac{1}{2}}(1-x)^{s_{3}-\frac{3}{2}} d x .
$$

Consequently

$$
\sigma^{-1} n^{-1} \widetilde{X}_{n} \stackrel{\mathcal{L}}{\rightarrow} X^{(1 / 2)},
$$

where $X^{(1 / 2)}$ has the unique distribution whose sth moment is given by $m_{s}$.

Proof sketch. We provide an outline of the proof, leaving the details to the reader. We claim that it is sufficient to show that

$$
\rho^{n} \tilde{\mu}_{n}^{[s]} T_{n}=\left[C_{s}+o(1)\right] n^{s-\frac{3}{2}},
$$

with $C_{0}=c, C_{1}=0$, and for $s \geq 2$,

$$
C_{s}=\frac{1}{b \sqrt{\rho}} \frac{\Gamma(s-1)}{\Gamma\left(s-\frac{1}{2}\right)} \sum_{\substack{s_{1}+s_{2}+s_{3}=s \\
s_{2}, s_{3}<s}}\left(\begin{array}{c}
s \\
s_{1}, s_{2}, s_{3}
\end{array}\right)\left(\frac{\sigma}{\sqrt{2 \pi}}\right)^{s_{1}} C_{s_{2}} C_{s_{3}} J_{s_{1}, s_{2}, s_{3}} .
$$

Indeed, defining $m_{s}:=\sigma^{-s} c^{-1} C_{s}$ and proceeding as in Theorem 4.2 yields the claim.

To show (4.26), we proceed by induction. The case $s=0$ is easily checked, and the case $s=1$ follows from (4.17). For $s \geq 2$ we use the induction hypothesis and approximation of sums by Riemann integrals in (4.25) to get

$$
\rho^{n}(n-1) T_{n} \tilde{r}_{n}^{[s]} \sim D_{s} n^{s-1}
$$

where

$$
D_{s}:=a_{1} \sum_{\substack{s_{1}+s_{2}+s_{3}=s \\
s_{2}, s_{3}<s}}\left(\begin{array}{c}
s \\
s_{1}, s_{2}, s_{3}
\end{array}\right)\left(\frac{\sigma}{\sqrt{2 \pi}}\right)^{s_{1}} C_{s_{2}} C_{s_{3}} J_{s_{1}, s_{2}, s_{3}} .
$$

Since we know a priori that $\tilde{r}^{[s]}(z)$ is amenable to singularity analysis it follows that [cf. [4.19)]

$$
\tilde{r}^{[s]}(z) \sim \Gamma(s) D_{s} Z^{-s}
$$

and completing the computations as in the proof of Proposition4.1 yields the proof of (4.26).

\section{One-Sided DESTRUCTION}

5.1. Expectation. We study equation (3.17) for the toll $t_{n}=n^{\alpha}$ with $\alpha \geq 0$ and start by establishing a singular expansion for the expectation $\mu^{[1]}(z)$. Since $\mu^{[0]}(z)=T(z)$, we have from (3.14) that

$$
{ }^{[1]}(z)=t(z) \odot\left[a_{1} z T^{\prime}(z) T(z)+a_{0} T^{2}(z)\right]
$$

which has already been considered in Section 4.1 In the remaining part of Section 5.1 we suppose now $\alpha \notin\left\{\frac{1}{2}, \frac{3}{2}, \ldots\right\} \cup\{0,1,2, \ldots\}$. (The complementary cases are covered in the proof of Theorem [5.1]) Then a compatible singular expansion for $r^{[1]}(z)$ is available at (4.9). This leads to the expansion (4.10) for $g^{[1]}(z)$ and consequently, using (2.7), to

$$
\frac{g^{[1]}(z)}{T(z)} \sim \frac{\Gamma\left(\alpha+\frac{1}{2}\right)}{2 \rho \sqrt{\pi}} Z^{-\alpha-1}+Z^{-\alpha-\frac{1}{2}} \mathcal{A}+Z^{-\alpha} \mathcal{A}+Z^{-1 / 2} \mathcal{A}+\mathcal{A}
$$


Integrating the last expression gives the singular expansion

$$
\int_{0}^{z} \frac{g^{[1]}(t)}{T(t)} d t \sim \frac{\Gamma\left(\alpha+\frac{1}{2}\right)}{2 \alpha \sqrt{\pi}} Z^{-\alpha}+Z^{-\alpha+\frac{1}{2}} \mathcal{A}+Z^{-\alpha+1} \mathcal{A}+\mathcal{A}+Z^{1 / 2} \mathcal{A}
$$

Now using (3.17), we obtain easily the desired expansion for $\mu^{[1]}(z)$ :

$$
\begin{aligned}
\mu^{[1]}(z) & =T(z) \int_{0}^{z} \frac{g^{[1]}(t)}{T(t)} d t+t_{1} T(z) \\
& \sim \frac{\tau \Gamma\left(\alpha+\frac{1}{2}\right)}{2 \alpha \sqrt{\pi}} Z^{-\alpha}+Z^{-\alpha+\frac{1}{2}} \mathcal{A}+Z^{-\alpha+1} \mathcal{A}+\mathcal{A}+Z^{1 / 2} \mathcal{A} .
\end{aligned}
$$

Via singularity analysis, we thus get the following expansion for the coefficients:

$$
\rho^{n}\left[z^{n}\right] \mu^{[1]}(z)=\rho^{n} \mu_{n}^{[1]} T_{n} \sim \frac{\tau \Gamma\left(\alpha+\frac{1}{2}\right)}{2 \sqrt{\pi} \Gamma(\alpha+1)} n^{\alpha-1}+n^{\alpha-\frac{3}{2}} \mathcal{N}+n^{\alpha-2} \mathcal{N}+n^{-3 / 2} \mathcal{N},
$$

which together with (2.2) yields the full asymptotic expansion

$$
\mu_{n}^{[1]} \sim \frac{\sigma \Gamma\left(\alpha+\frac{1}{2}\right)}{\sqrt{2} \Gamma(\alpha+1)} n^{\alpha+\frac{1}{2}}+n^{\alpha} \mathcal{N}+n^{\alpha-\frac{1}{2}} \mathcal{N}+\mathcal{N},
$$

with $\sigma$ defined at (2.3).

5.2. Higher moments and limiting distributions. We state the main result of this section:

Theorem 5.1. Let $\alpha \geq 0$. Define $\sigma:=\tau \sqrt{\frac{\Phi^{\prime \prime}(\tau)}{\Phi(\tau)}}$ and $\alpha^{\prime}:=\alpha+\frac{1}{2}$. Then, for toll function $t_{n}=n^{\alpha}$, the moments $\mu_{n}^{[s]}:=\mathbf{E} Y_{n}^{s}$ satisfy the following asymptotic expansion as $n \rightarrow \infty$ :

$$
\mu_{n}^{[s]}=\frac{s ! \sigma^{s}}{2^{s / 2}} \prod_{j=1}^{s} \frac{\Gamma\left(j \alpha^{\prime}\right)}{\Gamma\left(j \alpha^{\prime}+\frac{1}{2}\right)} n^{s \alpha^{\prime}}+O\left(n^{s \alpha^{\prime}-q}\right),
$$

with

$$
q:= \begin{cases}\frac{1}{2}-\epsilon & \text { if } \alpha \in\{0,1 / 2\} \\ \min \{\alpha, 1 / 2\} & \text { otherwise, }\end{cases}
$$

where $\epsilon>0$ is arbitrarily small. Thus the normalized random variable $Y_{n}$ converges weakly to a random variable $Y^{(\alpha)}$ :

$$
\sigma^{-1} n^{-\alpha^{\prime}} Y_{n} \stackrel{\mathcal{L}}{\rightarrow} Y^{(\alpha)},
$$

where $Y^{(\alpha)}$ has the unique distribution with (for $s \geq 1$ ) sth moment

$$
m_{s}=\frac{s !}{2^{s / 2}} \prod_{j=1}^{s} \frac{\Gamma\left(j \alpha^{\prime}\right)}{\Gamma\left(j \alpha^{\prime}+\frac{1}{2}\right)} .
$$

In particular when $\alpha=0$ (i.e., $t_{n} \equiv 1$ ), $\sigma n^{-1 / 2} Y_{n}$ converges weakly to a standard Rayleigh distributed random variable $Y^{(0)}$ with density

$$
f(y)=y e^{-y^{2} / 2}, \quad y \geq 0 .
$$

In this case the asymptotics of $\mu_{n}^{[s]}$ can be sharpened to

$$
\mu_{n}^{[s]}=\frac{s ! \sigma^{s} \sqrt{\pi}}{2^{s / 2} \Gamma\left(\frac{s+1}{2}\right)} n^{s / 2}\left[1+O\left(\frac{\log n}{\sqrt{n}}\right)\right] .
$$


Proof. We use induction on $s$. We begin with $\alpha>0$. Observe that it is sufficient to show that the generating functions $\mu^{[s]}(z)$ admit the asymptotic expansions (5.3) around their dominant singularities at $z=\rho$. Then, using singularity analysis, the claim follows. What we will show is that

$$
\mu^{[s]}(z)=\frac{s ! \sigma^{s-1} \tau}{2^{\frac{s+1}{2}}\left(s \alpha^{\prime}-\frac{1}{2}\right) \sqrt{\pi}} \gamma_{s} Z^{-s \alpha^{\prime}+\frac{1}{2}}+O\left(|Z|^{-s \alpha^{\prime}+\frac{1}{2}+q}\right),
$$

where

$$
\gamma_{s}:=\frac{\prod_{j=1}^{s} \Gamma\left(j \alpha^{\prime}\right)}{\prod_{j=1}^{s-1} \Gamma\left(j \alpha^{\prime}+\frac{1}{2}\right)} .
$$

First we consider $s=1$, where we immediately obtain from the full expansion (5.1) that (5.3) is true for all $\alpha \notin\left\{\frac{1}{2}, \frac{3}{2}, \ldots\right\} \cup\{1,2, \ldots\}$. If on the other hand $\alpha \in\left\{\frac{1}{2}, \frac{3}{2}, \ldots\right\} \cup\{1,2, \ldots\}$, then, repeating the computations of Section [5.1] it is easily seen that logarithmic terms appear in the expansion of $\mu^{[1]}(z)$. But apart from the case $\alpha=\frac{1}{2}$, they don't have an influence on the main term or on the asymptotic growth order of the second-order term. If $\alpha=\frac{1}{2}$, one observes that the general formula for the main term holds, but the bound for the remainder term is different: $O\left(\left|\log Z^{-1}\right|\right)$, not $O(1)$. Summarizing these cases, the expansion (5.3) holds for $s=1$.

Next we assume that (5.3) holds for all $1 \leq s_{2}<s$ with a given $s>1$. From (5.3) follows the expansion

$$
\partial_{z} \mu^{\left[s_{2}\right]}(z)=\frac{s_{2} ! \sigma^{s_{2}-1} \tau}{2^{\frac{s_{2}+1}{2}} \sqrt{\pi} \rho} \gamma_{s_{2}} Z^{-s_{2} \alpha^{\prime}-\frac{1}{2}}+O\left(|Z|^{-s_{2} \alpha^{\prime}-\frac{1}{2}+q}\right),
$$

which holds for all $1 \leq s_{2}<s$. Together with $\mu^{[0]}(z)=T(z)$ and $a_{1} \tau=1$, this gives the following singular expansion:

$$
\begin{aligned}
& T(z)\left(a_{1} z \partial_{z} \mu^{\left[s_{2}\right]}(z)+a_{0} \mu^{\left[s_{2}\right]}(z)\right)= \\
& \begin{cases}\frac{1}{2} b \rho^{1 / 2} Z^{-1 / 2}+O(1), & s_{2}=0 \\
\frac{s_{2} ! \sigma^{s_{2}-1} \tau}{2^{\left(s_{2}+1\right) / 2} \sqrt{\pi}} \gamma_{s_{2}} Z^{-s_{2} \alpha^{\prime}-\frac{1}{2}}+O\left(|Z|^{-s_{2} \alpha^{\prime}-\frac{1}{2}+q}\right), & 1 \leq s_{2}<s .\end{cases}
\end{aligned}
$$

Under the assumptions $s_{1}+s_{2}=s$ and $s_{2}<s$, we get via singularity analysis the expansion

$$
\begin{aligned}
\rho^{n}\left[z^{n}\right]\left(\begin{array}{c}
s \\
s_{1}
\end{array}\right) t^{\odot s_{1}}(z) \odot\left[T(z)\left(a_{1} z \partial_{z} \mu^{\left[s_{2}\right]}(z)+a_{0} \mu^{\left[s_{2}\right]}(z)\right)\right]= \\
\begin{cases}c n^{s \alpha-\frac{1}{2}}+O\left(n^{s \alpha-1}\right), & s_{2}=0 \\
\left(\begin{array}{c}
s \\
s_{1}
\end{array}\right) \frac{s_{2} ! \sigma^{s_{2}-1} \tau}{2^{\left(s_{2}+1\right) / 2} \sqrt{\pi}} \prod_{j=1}^{s_{2}} \frac{\Gamma\left(j \alpha^{\prime}\right)}{\Gamma\left(j \alpha^{\prime}+\frac{1}{2}\right)} n^{s \alpha+\frac{s_{2}-1}{2}}+O\left(n^{s \alpha+\frac{s_{2}-1}{2}-q}\right), & 1 \leq s_{2}<s .\end{cases}
\end{aligned}
$$

Thus under the assumptions given above, the dominant contribution to $r^{[s]}(z)$ is obtained when $s_{2}=s-1$ and $s_{1}=1$, giving the expansion

$$
\rho^{n}\left[z^{n}\right] r^{[s]}(z)=\frac{s ! \sigma^{s-2} \tau}{2^{s / 2} \sqrt{\pi}} \prod_{j=1}^{s-1} \frac{\Gamma\left(j \alpha^{\prime}\right)}{\Gamma\left(j \alpha^{\prime}+\frac{1}{2}\right)} n^{s \alpha^{\prime}-1}+O\left(n^{s \alpha^{\prime}-1-q}\right),
$$

which in turn yields the following singular expansion for $r^{[s]}(z)$ :

$$
{ }^{[s]}(z)=\frac{s ! \sigma^{s-2} \tau}{2^{s / 2} \sqrt{\pi}} \gamma_{s} Z^{-s \alpha^{\prime}}+O\left(|Z|^{-s \alpha^{\prime}+q}\right) .
$$


Immediately from (3.16) and (2.6) follow the expansions

$$
g^{[s]}(z)=\frac{s ! \sigma^{s-1} \tau}{2^{(s+1) / 2} \sqrt{\pi} \rho} \gamma_{s} Z^{-s \alpha^{\prime}-\frac{1}{2}}+O\left(|Z|^{-s \alpha^{\prime}-\frac{1}{2}+q}\right)
$$

and

$$
\frac{g^{[s]}(z)}{T(z)}=\frac{s ! \sigma^{s-1}}{2^{(s+1) / 2} \sqrt{\pi} \rho} \gamma_{s} Z^{-s \alpha^{\prime}-\frac{1}{2}}+O\left(|Z|^{-s \alpha^{\prime}-\frac{1}{2}+q}\right) .
$$

Integrating leads to

$$
\int_{0}^{z} \frac{g^{[s]}(t)}{T(t)} d t=\frac{s ! \sigma^{s-1}}{2^{(s+1) / 2} \sqrt{\pi}\left(s \alpha^{\prime}-\frac{1}{2}\right)} \gamma_{s} Z^{-s \alpha^{\prime}+\frac{1}{2}}+O\left(|Z|^{-s \alpha^{\prime}+\frac{1}{2}+q}\right) .
$$

Using (3.17) and (2.17), we obtain (5.3) and Theorem 5.1] is proved for $\alpha>0$.

The case $\alpha=0$ has already been proved in [15], where the distribution has been characterized by its moments. Therefore we describe only very briefly how to obtain this result with the present approach.

One need only show by induction the singular behavior

$$
\begin{aligned}
\mu^{[s]}(z) & \\
= & \begin{array}{ll}
\frac{\tau}{2} \ln Z^{-1}+\mathcal{A}+O\left(\left|Z^{1 / 2} \log Z^{-1}\right|\right), & s=1, \\
\sqrt{2} \sigma \tau Z^{-1 / 2}+O\left(\left|\log Z^{-1}\right|^{2}\right), & s=2, \\
\frac{\tau}{\sqrt{\pi}} \sigma^{s-1} 2^{(s-1) / 2} \Gamma\left(\frac{s}{2}+1\right) \Gamma\left(\frac{s-1}{2}\right) Z^{-(s-1) / 2}+O\left(\left|Z^{-\frac{s}{2}+1} \log Z^{-1}\right|\right), & s \geq 3 .
\end{array}
\end{aligned}
$$

The desired result then follows by applying singularity analysis and the duplication formula for the $\Gamma$-function.

To begin the proof of (5.5), first we remark that for $s=1$ one proceeds as in Section 5.1 and gets the full expansion

$$
\mu^{[1]}(z)=\frac{\tau}{2} \ln Z^{-1}+\mathcal{A}+\left(Z^{1 / 2} \log Z^{-1}\right) \mathcal{A}+Z^{1 / 2} \mathcal{A}+\left(Z \log Z^{-1}\right) \mathcal{A},
$$

which of course gives (5.5) in that case. Assuming that (5.5) holds for $1 \leq s_{2}<s$ with a given $s \geq 2$, we have the singular expansion

$$
\begin{aligned}
\partial_{z} \mu^{\left[s_{2}\right]}(z)=\frac{\tau}{\sqrt{\pi} \rho} \sigma^{s_{2}-1} 2^{\left(s_{2}-1\right) / 2} \Gamma\left(\frac{s_{2}}{2}+1\right) \Gamma & \left(\frac{s_{2}+1}{2}\right) Z^{-\left(s_{2}+1\right) / 2} \\
& +O\left(\left|Z^{-\frac{s_{2}}{2}} \log Z^{-1}\right|\right) .
\end{aligned}
$$

Under the restrictions $s_{1}+s_{2}=s$ and $s_{2}<s$, we obtain via singularity analysis the expansions

$$
\begin{aligned}
\rho^{n}\left[z^{n}\right]\left\{\left(\begin{array}{c}
s \\
s_{1}
\end{array}\right) t^{\odot s_{1}}(z)\right. & \left.\odot\left[T(z)\left(a_{1} z \partial_{z} \mu^{\left[s_{2}\right]}(z)+a_{0} \mu^{\left[s_{2}\right]}(z)\right)\right]\right\} \\
= & \left(\begin{array}{c}
s \\
s_{1}
\end{array}\right) \frac{\tau}{\sqrt{\pi}} \sigma^{s_{2}-1} 2^{\frac{s_{2}-1}{2}} \Gamma\left(\frac{s_{2}}{2}+1\right) n^{\frac{s_{2}-1}{2}}+O\left(n^{\frac{s_{2}}{2}-1} \log n\right)
\end{aligned}
$$

for $1 \leq s_{2}<s$. [For $s_{2}=0$, an expansion is already available at (4.7).] Under the given restrictions, the dominant contribution to $r^{[s]}(z)$ is obtained when $s_{2}=s-1$ and we obtain the following singular behavior of $r^{[s]}(z)$ :

$$
r^{[s]}(z)=\frac{\tau}{\sqrt{\pi}} s \sigma^{s-2} 2^{\frac{s}{2}-1} \Gamma\left(\frac{s+1}{2}\right) \Gamma\left(\frac{s}{2}\right) Z^{-s / 2}+O\left(\left|Z^{-\frac{s-1}{2}} \log Z^{-1}\right|\right) .
$$


We proceed with

$$
\frac{g^{[s]}(z)}{T(z)}=\frac{1}{\rho \sqrt{\pi}} s \sigma^{s-1} 2^{\frac{s-3}{2}} \Gamma\left(\frac{s+1}{2}\right) \Gamma\left(\frac{s}{2}\right) Z^{-\frac{s+1}{2}}+O\left(\left|Z^{-s / 2} \log Z^{-1}\right|\right),
$$

and, due to (3.17) and (2.1), integrating gives (5.5) for $s \geq 2$ and completes the proof.

\section{REFERENCES}

[1] W. E. Boyce and R. C. DiPrima. Elementary differential equations and boundary value problems. John Wiley \& Sons, 4th edition, 1986.

[2] P. Chassaing and R. Marchand. In preparation.

[3] P. Chassaing and R. Marchand. Merging costs for the additive Marcus-Lushnikov process, and Union-Find algorithms, arXiv:math.PR/0406094.

[4] J. A. Fill, P. Flajolet, and N. Kapur. Singularity analysis, Hadamard products, and tree recurrences. J. Comput. Appl. Math., 174(2):271-313, 2005, arXiv:math.CO/0306225.

[5] J. A. Fill and N. Kapur. An invariance principle for simply generated families of trees. Technical report, Department of Mathematical Sciences, The Johns Hopkins University, 2003.

[6] J. A. Fill and N. Kapur. Limiting distributions for additive functionals on Catalan trees. Theoret. Comput. Sci., 326:69-102, 2004, arXiv:math.PR/0306226.

[7] P. Flajolet. Singularity analysis and asymptotics of Bernoulli sums. Theoret. Comput. Sci., 215(1-2):371-381, 1999.

[8] P. Flajolet and A. Odlyzko. Singularity analysis of generating functions. SIAM J. Discrete Math., 3(2):216-240, 1990.

[9] P. Flajolet and R. Sedgewick. Analytic combinatorics. Book in preparation. Draft available at http://algo.inria.fr/flajolet/Publications/books.html

[10] S. Janson. Random cutting and records in deterministic and random trees, 2004. Available at http://www.math.uu.se/ `svante/papers/index.html

[11] D. E. Knuth and B. Pittel. A recurrence related to trees. Proc. Amer. Math. Soc., 105(2):335349, 1989.

[12] D. E. Knuth and A. Schönhage. The expected linearity of a simple equivalence algorithm. Theoret. Comput. Sci., 6(3):281-315, 1978.

[13] A. Meir and J. W. Moon. Cutting down random trees. J. Austral. Math. Soc., 11:313-324, 1970.

[14] A. Meir and J. W. Moon. On the altitude of nodes in random trees. Canad. J. Math., 30(5):997-1015, 1978.

[15] A. Panholzer. Cutting down very simple trees, 2003. Preprint.

[16] A. Panholzer. Non-crossing trees revisited: cutting down and spanning subtrees. In Discrete random walks (Paris, 2003), Discrete Math. Theor. Comput. Sci. Proc., AC, pages 265-276 (electronic). Assoc. Discrete Math. Theor. Comput. Sci., Nancy, 2003.

E-mail address, James Allen Fill: jimfill@jhu.edu

E-mail address, Nevin Kapur: nkapur@cs.caltech.edu

E-mail address, Alois Panholzer: Alois.Panholzer@tuwien.ac.at

URL, James Allen Fill: http://www.ams.jhu.edu/ fill/

$U R L$, Nevin Kapur: http://www.cs.caltech.edu/ nkapur/

URL, Alois Panholzer: http://info.tuwien.ac.at/panholzer/

(James Allen Fill) Applied Mathematics and Statistics, The Johns Hopkins University, 3400 N. Charles St., Baltimore MD 21218-2682

(Nevin Kapur) Computer Science, California Institute of Technology, MC 256-80, 1200 E. California Blvd., Pasadena CA 91125

(Alois Panholzer) Institut für Diskrete Mathematik und Geometrie, Technische Universität Wien, Wiedner Hauptstrasse 8-10/104, A-1040 Wien, Austria 